\title{
Etnomodelagem como um movimento de globalização nos contextos da Etnomatemática e da Modelagem
}

Milton Rosa

\section{Resumo}

A sociedade A conscientização sobre o desenvolvimento dos conhecimentos local (êmico) e global (ético) é um objetivo importante para a condução de investigações em Etnomodelagem. O conhecimento local é relevante para uma compreensão intuitiva e empática das ideias, procedimentos e práticas matemáticas desenvolvidas no decorrer da história. Enquanto o conhecimento global é importante para as comparações transculturais que exigem categorias analíticas padronizadas que possibilitam a comunicação entre sistemas de conhecimento matemático distintos. A glocalização utiliza os conhecimentos matemáticos local e global por meio do diálogo simétrico e da interação, pois busca a tradução entre esses sistemas. O objetivo desse artigo teórico é discutirmos as abordagens matemáticas local, global e glocal que são necessárias para o desenvolvimento de pesquisas em Etnomodelagem.

Palavras-chave: Abordagem Global, Abordagem Local, Etnomodelagem, Glocalização. 


\title{
Ethnomodelling as glocalization movement in the contexts of the Mathematics and Modelling
}

Milton Rosa

\begin{abstract}
The awareness about the development of both local (emic) and global (etic) knowledge is an important goal for the conduction of Ethnomodelling investigations. Local knowledge (emic) is relevant for an intuitive and empathic understanding of mathematical ideas, procedures, and practices developed throughout history. While global knowledge (etic) is important for the achievement of cross-cultural comparisons that demand standard analytical units and categories to enable communication between distinct mathematical systems. Glocalization uses both local and global mathematical knowledge by means of symmetrical dialogue and interaction through translation between these systems. Thus, the main objective of this theoretical article is to discuss the local, global, and glocal mathematical approaches that are necessary for the development of ethnomodeling investigations.
\end{abstract}

Keywords: Global Approach, Local Approach, Ethnomodelling, Glocalization. 


\section{Considerações Iniciais}

As pessoas sempre buscaram inteirar-se de outras culturas para compartilhar ou trocar conhecimentos e informações. Esse contato entre culturas propiciou uma troca de capital cultural ${ }^{6}$ que pode ter influenciado e enriquecido a construção sociocultural de comunidades distintas durante essa convivência. Dentre esses conhecimentos, a matemática, muitas vezes, estava escondida nas ideias, nos procedimentos e nas práticas diversas realizadas no cotidinao.

Ressalta-se que uma das consequências desse fenômeno de interação entre culturas diferentes foi o domínio de um consenso generalizado que sustentou a supremacia dos sistemas matemáticos ocidentais (global) ao excluir a maioria das outras tradições locais (ROSA; OREY, 2015), propiciando a destruição ou o desgaste de outras culturas. Por exemplo, Powell e Frankenstein (1997) afirmam que as fundações gregas da civilização europeia foram constituídas com embasamento na cultura egípcia.

Nesse contexto, as formas dominantes, imperialistas e colonialistas de valores culturais influenciaram a maneira pela qual os membros de grupos culturais distintos compreendem as ideias, os procedimentos e as práticas matemáticas que foram desenvolvidas no decorrer da história. Dessa maneira, Rosa e Orey (2015) argumentam que, no currículo escolar, os métodos de resolução de problemas estão baseados nas tradições matemáticas mediterrâneas e, com raríssimas exceções, nos conhecimentos desenvolvidos pelas culturas ocidentais.

Dessa maneira, a maioria dos exemplos utilizados no processo de ensino e aprendizagem em Matemática é derivada de culturas anglo-saxônicas e europeias, sem a participação latina ou africana no desenvolvimento dessa ação educativa. Ressalta-se que os métodos de resolução de problemas e de matematização foram introduzidos, principalmente, pelas visões de mundo europeias sobre o conhecimento matemático (ROSA; OREY, 2015).

Diferentes culturas, contudo, contribuíram para o desenvolvimento das ideias, procedimentos e práticas matemáticas que enriqueceram os conceitos tradicionais desse campo de estudo. É importante enfatizar que essa interação também colaborou, timidamente, para iniciar um movimento de valorização de conhecimentos matemáticos originados nas culturas locais (D’AMBROSIO, 2006a).

Por meio das pesquisas sobre as práticas matemáticas desenvolvidas pelos membros de grupos culturais distintos, os pesquisadores se inteiraram das ideias, dos procedimentos e das práticas matemáticas diferentes daquelas estudadas em instituições acadêmicas. Esse

${ }^{36} \mathrm{O}$ capital cultural está relacionado com os conhecimentos, as experiências e as conexões que os membros de grupos culturais distintos desenvolvem no decorrer de suas vidas. Esse capital também pode ser considerado como uma relação social que está inserida em um sistema de trocas, que inclui o conhecimento cultural acumulado, conferindo, assim, empoderamento para os indivíduos que o possuem (ROSA, 2010). 
conhecimento matemático pode ser traduzido por meio da Etnomodelagem, pois é um processo que envolve uma ação pedagógica holística que incorpora os conceitos de globalização e localização (ROSA; OREY, 2010). Esse processo expande a conceituação da interculturalidade ${ }^{37}$ que busca a apreciação, o respeito e a valorização do conhecimento matemático desenvolvido em outras culturas.

Rosa (2010) argumentou que, nesse processo, é necessário que os membros desses grupos encontrem um equilíbrio para garantir que as ideias e os procedimentos matemáticos locais não sejam sufocados por noções e práticas matemáticas globais, pois existe a necessidade de que haja um reconhecimento do saber/fazer matemático desenvolvido por essas pessoas. Por conseguinte, de acordo com D’Ambrosio (1993), um dos principais objetivos da Etnomatemática é considerar os modos de saber e de fazer das várias culturas para reconhecer como e porque grupos de indivíduos, organizados como famílias, comunidades, profissões, tribos, nações e povos, executam as suas práticas de natureza Matemática, como, por exemplo, contar, medir, comparar, classificar.

Essa abordagem possibilita a elaboração de ações pedagógicas originadas no contexto sociocultural dos alunos, que são desenvolvidas com a utilização da Etnomodelagem. Esse contexto propicia a valorização das ideias e dos procedimentos matemáticos locais, bem como o reconhecimento de práticas matemáticas globais que objetivam a valorização e o respeito aos valores culturais e os conhecimentos adquiridos pelos alunos em sua convivência em sociedade.

Esse equilíbrio pode ser alcançado por meio da glocalização $0^{38}$, que é a capacidade que os membros de grupos culturais distintos desenvolvem para valorizar os traços culturais ${ }^{39}$ globais que podem enriquecer a própria cultura, resistindo, contudo, às características que são estranhas à sua identidade cultural (FRIEDMAN, 2000). Por exemplo, D'Ambrosio (2006b) afirma que "toda cultura está sujeita aos encontros inter e intraculturais40" (p. 76), possibilitando que esse dinamismo cultural41, embora diferente, possa ser desfrutado e celebrado com alteridade.

37Os encontros interculturais descrevem as experiências trocadas entre pelo menos duas pessoas que são culturalmente diferentes em termos significativos ou que tenham origens culturais distintas, como, por exemplo, regionais, sociais, linguísticas, econômicas, políticas, étnicas ou religiosas (ROSA, 2010). ${ }^{38} \mathrm{~A}$ glocalização é um conceito cunhado no campo dos negócios que significa a produção de mercadorias para o mercado global, contudo, personalizados para se adequarem às culturas e aos gostos locais (ROBERTSON, 1995).

$39 \mathrm{O}$ traço cultural é socialmente aprendido e apreendido das crenças, dos valores, das tradições, dos símbolos e dos significados que os membros de uma cultura específica adquirem no decorrer de sua história, de geração em geração (ROSA, 2010).

$4^{\circ} \mathrm{Os}$ encontros intraculturais descrevem as experiências entre pelo menos duas pessoas que são da mesma cultura ou que possuem antecedentes culturalmente semelhantes (ROSA, 2010).

${ }^{41} \mathrm{O}$ dinamismo cultural refere-se à troca de saberes e fazeres entre sistemas de conhecimentos distintos que possibilitam aos membros de culturas distintas a exploração ou a adaptação ao mundo que os rodeia (ROSA; OREY, 2017a). 
Nesse contexto, ressalta-se que as características diversas e diferenciadas das ideias e procedimentos matemáticos podem ser encontrados quando os pesquisadores investigam o conhecimento matemático desenvolvido pelos membros de grupos culturais distintos no decorrer da história. No entanto, frequentemente, a compreensão (ética, global) desses observadores externos sobre os traços culturais pode ser entendida como uma interpretação equivocada da natureza das práticas matemáticas que foram desenvolvidas e modeladas para que esses membros pudessem resolver as situações-problema enfrentadas diariamente.

Assim, a multiplicidade de culturas, cada uma com um sistema próprio de conhecimentos compartilhados e um conjunto compatível de comportamentos e valores específicos, propiciou o desenvolvimento da dinâmica cultural dos encontros (D’AMBROSIO, 1993). Esse contexto possibilitou uma progressiva familiaridade com a diversidade de conhecimentos que foram aprimorados pela humanidade, inclusive o matemático, que originou uma crescente necessidade para o desenvolvimento de um campo de pesquisa que pudesse modelar os fenômenos que ocorrem em diversos contextos culturais.

Essa perspectiva utilizou os métodos de: Resolução de Problemas, Categorização e Estruturas conceituais para expandir o entendimento e a compreensão dos fenômenos econômicos, políticos e ambientais por meio da elaboração de modelos durante a condução do processo de Modelagem Matemática. Geralmente, os modelos podem ser considerados como conjuntos de "símbolos e relações matemáticas que representam de alguma forma o objeto estudado" (BASSANEZI, 2002, p. 20).

Vários modelos são elaborados a partir dos dados obtidos com estudos etnomatemáticos, que propõem a redescoberta de sistemas de conhecimento matemático que foram desenvolvidos pelos membros de grupos culturais distintos. Esses modelos são denominados de etnomodelos, que são unidades de informação que compõem a representação dos sistemas retirados da realidade desses membros para representar os fenômenos cotidianos que traduzem as práticas matemáticas culturais locais (ROSA; OREY, 2015).

Nesse sentido, os etnomodelos são representações que podem ser consideradas como artefatos culturais ${ }^{42}$ que são utilizados para o entendimento e a compreensão da realidade dos membros de grupos culturais específicos (ROSA; OREY, 2010). Nesse processo, há o reconhecimento de que os fundamentos da Etnomodelagem diferem dos métodos tradicionais utilizados na Modelagem, pois utilizam a noção de global, local e glocal para traduzirem as ideias, os procedimentos e as práticas matemáticas desenvolvidas em sistemas de conhecimento matemático distintos.

${ }^{42}$ Os artefatos culturais são objetos criados pelos membros de grupos culturais distintos que fornecem pistas e informações sobre os seus criadores e usuários (D'AMBROSIO, 1993).

ISSN 2526-2882 
Assim, nesse artigo objetivamos compartilhar as nossas reflexões sobre as pesquisas desenvolvidas em Etnomodelagem em contextos diversos. Desse modo, discutimos as abordagens local, global e glocal que são necessárias para o desenvolvimento de investigações em Etnomodelagem. Esperamos que esse ponto de vista possa contribuir para uma compreensão holística (glocal) das ideias, dos procedimentos e das práticas matemáticas desenvolvidas pelos membros de grupos culturais distintos.

\section{Etnomatemática, Modelagem e Etnomodelagem}

Os primeiros modelos devem ter sido suscitados pela necessidade da sobrevivência dos membros de grupos culturais distintos, sendo que se constituíram a partir de abstrações matemáticas de situações cotidianas (ROSA; OREY, 2010). Nessa perspectiva, a Etnomatemática utiliza os etnomodelos retirados da realidade sociocultural desses membros como um processo que incorpora os códigos, os símbolos e as técnicas originadas em contextos distintos, em conjunto com a linguagem formalizada da matemática por meio da Etnomodelagem. Esses etnomodelos possibilitam que os membros de grupos culturais distintos assumam posicionamentos críticos e reflexivos com relação à natureza do objeto estudado (ROSA; OREY, 2010).

De acordo com essa perspectiva, Rosa e Orey (2013) afirmam que a Etnomodelagem está relacionada ao processo de elaboração de problemas e questões que se originam a partir de situações reais (sistemas) para representar uma imagem do matema ${ }^{43}$. Essa perspectiva forma essencialmente uma análise crítica e reflexiva para a geração e a produção do conhecimento (criatividade), desenvolvendo o processo intelectual de sua produção (história), bem como os mecanismos sociais de institucionalização do conhecimento e a sua transmissão (educação).

Assim, os membros de grupos culturais distintos estão permanentemente recebendo informações da realidade, processando-as e realizando ações para transformá-la. Contudo, embora imersos em uma mesma realidade global, os mecanismos para o recebimento dessas informações são diferentes (D'AMBROSIO, 2006a). De acordo com Rosa e Orey (2017a), é necessário destacar que os membros desses grupos criam, desenvolvem, acumulam e processam as informações presentes na realidade em modos diversos e, como consequência, por causa dessas diferentes perspectivas, as suas necessidades de sobrevivência e transcendência também se diversificam nesses contextos.

Desse modo, a análise desse processo possibilita que a modelagem seja conduzida a partir de um contexto sociocultural com a identificação do conhecimento dos alunos a fim de

$43 \mathrm{O}$ termo matema está relacionado ao processo holístico da compreensão e do desenvolvimento das ideias, dos procedimentos e das práticas matemáticas originadas em contextos socioculturais distintos (ROSA; OREY, 2017a). 
instigar a sua capacidade para a elaboração de modelos matemáticos em diferentes ambientes e aplicações (D’AMBROSIO, 1993). Similarmente, o processo de Etnomodelagem utiliza os contextos socioculturais para motivar os alunos por meio da realização de diálogos simétricos 44 .

A Etnomodelagem também ressalta a compreensão das práticas matemáticas desenvolvidas em contextos distintos, em especial, na compreensão de como certo conhecimento se originou em determinados grupos culturais. E, ainda, como o encontro entre culturas distintas envolve a realização de diálogos que se interpenetram, pois são realizados em diferentes níveis intraculturais. Nesse contexto, essa alteridade emergente 45 frequentemente utiliza as traduções entre sistemas de conhecimentos matemáticos distintos. De acordo com Iser (1994), essa utilização tem como objetivo a valorização da alteridade sem submetê-la, contudo, às noções pré-concebidas de valores e comportamentos externos ao próprio grupo cultural.

Por exemplo, D’Ambrosio (2006b) argumenta que há necessidade de que esses encontros sejam examinados de maneiras diversas, possibilitando, assim, as interações e as influências, bem como o exame de temáticas em uma base comparativa por meio da tradução entre sistemas de conhecimento diversos. Iser (1994) argumenta que a tradução é um conceito relevante para a compreensão de encontros entre diferentes culturas e, também, para a compreensão das interações que ocorrem dentro de grupos culturais distintos.

\section{Três abordagens culturais para a Etnomodelagem}

O desafio que os pesquisadores têm para lidar com as conexões entre a Matemática e a Cultura é a aplicação de procedimentos metodológicos que os auxiliem no entendimento das ideias e dos procedimentos matemáticos vinculados ao próprio contexto, sem viabilizar que a sua formação influencie na visão de mundo e no background dos outros. A esse respeito, é necessário enfatizar que os membros de grupos culturais distintos desenvolvem e desenvolveram a própria interpretação das práticas matemáticas locais (êmica) em oposição à interpretação global (ética) dos observadores externos sobre essas atividades.

Por conseguinte, é importante rever a origem das ideias, dos procedimentos e das práticas matemáticas exclusivamente europeia, pois estão fundamentadas em suposições e valores filosóficos vigorosamente endossados pela ciência ocidental. Se de um lado, existe a noção de que as práticas matemáticas são diversas e de que a identidade cultural está

44No diálogo simétrico ocorre a socialização das ideias e do conhecimento tacitamente adquirido, que pode gerar uma mudança comportamental nos membros de grupos culturais distintos por meio do desenvolvimento de ações transformadoras na sociedade (FREIRE, 2002).

45A alteridade é derivada do termo latino alter, que está relacionado com a qualidade de ser diferente, sendo considerada como um princípio filosófico de trocar a própria perspectiva de ser pela dos outros para se colocar em seu lugar (ROSA; OREY, 2017a) 
relacionada ao desenvolvimento dos membros de grupos culturais distintos e, por outro, que todas as práticas matemáticas são as mesmas em todos os lugares e que todos os seus objetivos e técnicas são igualmente aplicáveis pelos membros de todos os grupos culturais e que, se diferirem, são consideradas inferiores, rudimentares e simplistas.

Um objetivo importante da Etnomodelagem (sugiro que o termo seja Etnomodelagem) é desafiar os modelos teóricos existentes tanto em seus pressupostos de universalidade matemática quanto em suas reivindicações de adequação descritiva, preditiva e explicativa. Um segundo objetivo é compreender, explicar e entender a diversificação existente de ideias, procedimentos e práticas matemáticas que variam em culturas distintas, principalmente, com relação à raça, à etnia, ao gênero e às outras características socioculturais.

Nesse direcionamento, nas pesquisas em Etnomodelagem, é possível identificar três abordagens que podem auxiliar os pesquisadores a investigarem, estudarem e compreenderem as ideias, os procedimentos e as práticas matemáticas desenvolvidas pelos membros de grupos culturais distintos:

1) Global (ética): é a visão dos observadores externos, de fora, sobre as crenças, os costumes e o conhecimento matemático desenvolvido pelos membros de grupos culturais distintos. A globalização reforça a abordagem utilitarista da matemática escolar predominante nas escolas. Essa abordagem propiciou a transmissão de ideologias matemáticas difusas, bem como a noção de que a matemática escolar é uma força cultural homogeneizadora, um filtro para a manutenção do status e um instrumento de poder (ROSA; OREY, 2017b). Nessa abordagem, os pesquisadores comparativistas descrevem as semelhanças de procedimentos matemáticos desenvolvidos em culturas distintas. De acordo com Sue e Sue (2003), esses membros são considerados como culturalmente universais.

2) Local (êmica): é a visão dos membros de grupos culturais distintos sobre a própria cultura e crenças e, também, sobre os próprios costumes e conhecimento matemático. O conhecimento local é importante porque foi valorizado, testado e validado dentro do próprio contexto local. Atualmente, reconhece-se a importância das contribuições locais para o desenvolvimento do conhecimento matemático, pois desenvolve uma estrutura sociocultural a partir da qual esses membros podem entender e interpretar o mundo ao seu redor (BARBER, 2004). Nessa abordagem, os membros desses grupos descrevem a sua cultura em seus próprios termos. De acordo com Sue e Sue (2003), esses membros são considerados como culturalmente específicos.

3) Glocal (êmico-ético): a glocalização representa uma interação contínua entre a globalização e a localização, pois oferece a perspectiva de que ambas as abordagens são elementos importantes de um mesmo fenômeno. Essa abordagem possibilita agregar, misturar e adaptar duas abordagens complementares, na qual um dos 
componentes deve abordar a cultura local e os seus sistemas de valores e práticas (KHONDKER, 2004). Nesse contexto, é necessário trabalhar com diferentes ambientes culturais para que os membros de grupos culturais distintos atuem como etnógrafos para que possam descrever as ideias, os procedimentos e as práticas matemáticas desenvolvidas em outras culturas (D’AMBROSIO, 2006b).

Essas abordagens mostram que existe a necessidade de focalizar, primeiro, no conhecimento local e, em seguida, buscar uma minimização das influências globais, pois os membros de grupos culturais distintos estão enraizados em suas próprias tradições locais, mas também estão equipados com o conhecimento global, que possibilita o desenvolvimento de uma espécie de globalização localizada (CHENG, 2005) e vice-versa das práticas matemáticas.

De acordo com esse contexto, é preciso que os pesquisadores se conscientizem sobre a universalidade cultural (global) imposta ao conhecimento matemático enquanto assumem as técnicas, os procedimentos e as estratégias de seu relativismo cultural. Assim, esses pesquisadores buscam vincular o conhecimento matemático universal (global) com os saberes e fazeres matemáticos específicos (local), evitando enfrentar o clássico dilema entre essas duas abordagens, que conflitam com os pressupostos da Etnomodelagem.

Então, uma sugestão para lidar com esse suposto dilema é a utilização da abordagem dialógica, que combina ambas as abordagens: global e local, que possibilitam a familiarização dos membros de grupos culturais distintos com as diferenças culturais encontradas em contextos diversos. Assim, essas abordagens são complementares, pois propiciam o aprofundamento da compreensão de questões socioculturais que são relevantes para as investigações em Etnomodelagem (ROSA; OREY, 2013).

Nesse direcionamento, de acordo com Anderson (2007), a abordagem local procura entender a cultura em sua dinâmica interna e nos relacionamentos influenciados pelos entornos cultural, social, político, econômico e ambiental enquanto a abordagem global é comparativa, contrastante e transcultural, pois busca compreender e explicar as culturas conforme o ponto de vista de observadores externos. Para Rosa e Orey (2013), a Etnomodelagem possibilita o delineamento de formas de sinergia entre essas duas abordagens.

\section{Etnomodelagem e suas Confluências}

Os resultados de estudos mostram a utilização de ideias e procedimentos matemáticos sofisticados, que incluem as estruturas arquitetônicas, os conceitos de simetria, as práticas de confecção de artefatos e os princípios geométricos encontrados nos artesanatos, que são desenvolvidos pelas culturas locais (EGLASH et al, 2006; OREY, 2000; ROSA; OREY, 2013). 
Essas práticas estão relacionadas com a utilização de uma variedade de procedimentos matemáticos empregados na confecção de artefatos culturais, que incluem as relações numéricas encontradas nas atividades matemáticas universais de medição, cálculo, jogos, adivinhação, navegação, astronomia e modelagem (BISHOP, 1988). Essa vitalidade local libera um empoderamento cultural vigoroso que é reforçado pela participação plena e contínua de seus membros na comunidade e, simultaneamente, a sua atuação no mundo global (D’AMBROSIO, 2006b).

Nesse contexto, Rosa e Orey (2010) argumentam que a Etnomodelagem possibilita o desenvolvimento de uma ação pedagógica que utiliza a tradução de situações-problema relacionadas com as ideias, os procedimentos e as práticas matemáticas enraizadas em culturas diversas. Essa abordagem propicia a condução de investigações dinâmicas que incorporam os fenômenos culturalmente universais e específicos no desenvolvimento de ações pedagógicas inclusivas no processo de ensino e aprendizagem em Matemática.

Assim, a tradução é utilizada para descrever o processo de elaboração de etnomodelos em culturas locais, que podem ter representações em outros sistemas de conhecimento matemático, incluindo, o acadêmico (ROSA; OREY, 2010). Por exemplo, a representação dos padrões têxteis indígenas podem ter relações com as classificações simétricas cristalográficas utilizadas nas práticas matemáticas globais (EGLASH et al., 2006).

Por outro lado, a Etnomatemática pode utilizar a Modelagem para estabelecer as relações encontradas entre os marcos conceituais locais e as ideias matemáticas embutidas em inúmeros esquemas e desenhos globais. Essa relação é definida como Etnomodelagem, que pode ser considerada como a utilização de atos de tradução entre sistemas de conhecimento matemático distintos (ROSA. OREY, 2017b).

Por exemplo, a figura 1 mostra o conhecimento que as rendeiras do nordeste brasileiro utilizam para tecer os padrões formados por ideias e procedimentos matemáticos que estão desassociados dos princípios geométricos tradicionais, mas que pode ser modelado por meio da utilização de etnomodelos na condução do processo da Etnomodelagem.

Figura 1: Padrões de rendas geométricas

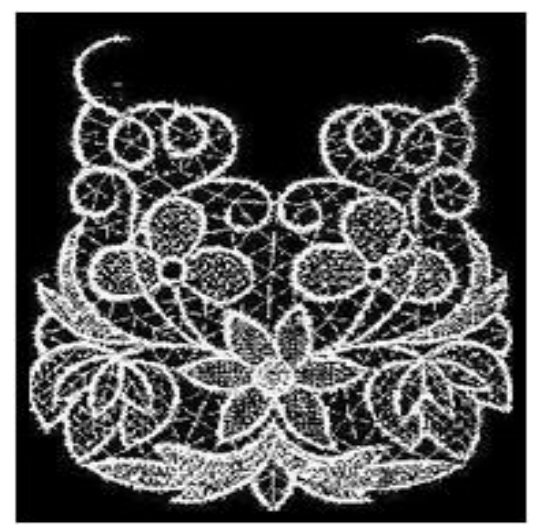

Fonte: Rosa e Orey (2013)

ISSN 2526-2882

* $* 7 *$ 
A Etnomodelagem considera os diversos processos interpretativos que auxiliam no desenvolvimento do conhecimento matemático, incluindo a interatividade, a coletividade e a criatividade, que se originam na confluência entre a Modelagem Matemática, a Etnomatemática e a Antropologia Cultural.

Esse contexto possibilita o desenvolvimento da Etnomodelagem através da tradução de ideias, procedimentos e práticas matemáticas por meio da elaboração de etnomodelos que se relacionam com o conhecimento matemático específico desenvolvido pelos membros de grupos culturais distintos. Por conseguinte, Rosa e Orey (2017b) afirmam que a Etnomatemática acrescenta as perspectivas culturais ao processo de modelagem. A figura 2 mostra a Etnomodelagem como a confluência entre três campos de conhecimento: a Modelagem Matemática, a Antropologia Cultural e a Etnomatemática.

Figura 2: Etnomodelagem como a confluência entre três campos do conhecimento

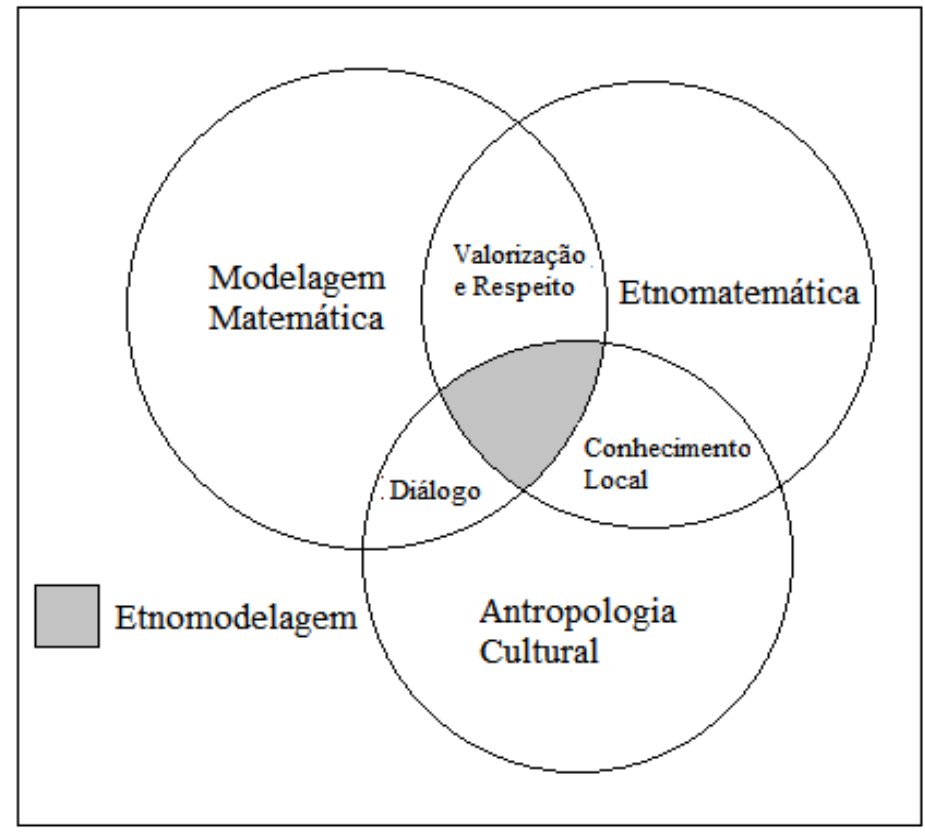

Fonte: Rosa e Orey (2013)

Nesse processo, a confluência entre a Modelagem Matemática e a Etnomatemática se relaciona com o respeito e a valorização dos saberes e tradições tácitas ${ }^{46}$ desenvolvidas pelos membros de grupos culturais distintos, permitindo que avaliem e traduzam as situaçõesproblema cotidianas por meio da elaboração de etnomodelos originados em diferentes contextos (ROSA; OREY, 2017b).

Portanto, torna-se necessário utilizar os contextos socioculturais, as realidades, as motivações e os interesses dos alunos por meio da desvinculação da mera aplicação de um conjunto de valores curriculares externos e, muitas vezes, de atividades descontextualizadas,

${ }^{46}$ As tradições tácitas estão baseadas nas emoções, experiências, introspecções, intuições, observações e informações internalizadas, que são desenvolvidas por meio da compreensão dos fenômenos que são desencadeadas no cotidiano (ROSA; OREY, 2013). 
para possibilitar o desenvolvimento de um diálogo simétrico entre a Modelagem Matemática e a Antropologia Cultural.

Essa abordagem propicia o fortalecimento de uma transitividade crítica que lida com o desenvolvimento de uma consciência reflexivamente transitiva caracterizada pelo desenvolvimento de uma educação dialógica e ativa, que é direcionada para a responsabilidade social e política. Esse processo se caracteriza pela profundidade e compreensão na resolução e interpretação dos problemas enfrentados no cotidiano (FREIRE, 2002).

Nesse contexto, o conhecimento matemático local tem origem na confluência entre a Etnomatemática e a Antropologia cultural, que ocorre quando os membros de grupos culturais distintos utilizam esse conhecimento para resolver as situações-problemas enfrentadas em seus próprios contextos histórico, social, cultural e natural por meio da elaboração de etnomodelos.

Portanto, na Etnomodelagem, o conhecimento matemático global deve ser reinventado e adaptado às realidades locais. Além disso, uma localização efetiva requer a promoção do conhecimento matemático por meio da globalização. Essa abordagem tem como objetivo tornar os conhecimentos matemáticos disponíveis em termos local e global por meio de um processo dialógico que está sujeito às transformações propiciadas pelo dinamismo cultural.

Desse modo, no encontro entre culturas distintas por meio da glocalização, as práticas são transformadas localmente ao mesmo tempo em que se difundem globalmente (LATOUR, 1993). Por conseguinte, a Etnomodelagem propicia o desenvolvimento de várias introspecções sobre o processo de glocalização, que inclui a interação entre as dimensões política, social, cultural, econômica e ambiental do trabalho institucional no processo de internacionalização das práticas matemáticas locais.

\section{Glocalização como uma Abordagem Transformadora para a Etnomodelagem}

Historicamente, os membros de grupos culturais distintos sempre contataram outras culturas. Para Iser (1994), em alguns casos, esses encontros buscaram um entendimento mútuo em termos da própria compreensão cultural, bem como em termos da especificidade do conhecimento desenvolvido pelos membros de outros grupos culturais. Portanto, D'Ambrosio (2006b) argumenta que como "resultado desses encontros, nenhuma cultura é estática e definitiva" (p. 76). Nesse sentido, é necessário apresentar uma abordagem alternativa às visões hegemônicas da globalização (ético) argumentando por uma contextualização mediada pela localização (êmico).

Então, de acordo com o ponto de vista da glocalização, a Etnomodelagem expressa as relações dialógicas que ocorrem entre as ideias, os procedimentos e as práticas matemáticas locais e globais. Esse diálogo proporciona o desenvolvimento do conhecimento matemático 
glocal47 que tem o potencial de gerar sinergias entre a globalização e a localização. Por exemplo, para Kloos (2000), a glocalização pode encorajar a criação de ambientes sinérgicos de relações interdependentes, reflexivas e coemergentes entre os processos global e local para o desenvolvimento do conhecimento matemático glocal.

Dessa maneira, Rosa e Orey (2018) argumentam sobre a importância de que as práticas matemáticas globais se adaptem às culturas locais e vice-versa. Esse contato do conhecimento matemático local com outros sistemas externos de conhecimento provoca o desenvolvimento do dinamismo cultural que ocorre durante os encontros entre culturas diversas. Esse contexto mostra que a glocalização do conhecimento matemático pode auxiliar na conscientização de que a objetividade e a subjetividade, a globalização e a localização e a universalização e a especificidade coexistem lado a lado (ROBERTSON, 1995) no desenvolvimento das ideias, procedimentos e práticas matemáticas.

Portanto, na Etnomodelagem, a glocalização propicia o desenvolvimento de uma base teórica para a incorporação de sistemas de conhecimento matemático advindos de práticas culturais locais, associando-os aos sistemas decorrentes de múltiplas visões de mundo. Por exemplo, os procedimentos e as práticas matemáticas globalizadas podem surgir das ideias e noções matemáticas localizadas e vice-versa.

Consequentemente, a glocalização pode ser considerada como um diálogo entre os sistemas de conhecimento matemático global e local, que se interagem e se complementam por meio da tradução entre essas abordagens, que são interdependentes e mutuamente constitutivas, que visa explicar como os membros desses grupos experimentam e vivenciam o mundo em termos socioculturais multiescalares (ROSA; OREY, 2017b).

Essa complementaridade é essencial devido a onipresença global da Matemática e da relevância das especificidades de suas expressões locais. Então, a Etnomodelagem se torna um produto sociocultural para o estudo da expansividade do conhecimento matemático por meio da glocalização. Nesse processo, é importante preservar a diversidade cultural do conhecimento matemático por meio das "maneiras pelas quais as formas se tornam separadas das práticas existentes e se recombinam em novas formas e em novas práticas” (ROWE; SCHELLING, 1991, p. 231).

De acordo com Friedman (2000), a glocalização é um processo pelo qual culturas distintas combinam as ideias, os procedimentos e as práticas diversas com as suas próprias tradições, pois visa capturar a dinâmica local-para-global-para-local. Contudo, é importante

47O conhecimento matemático glocal promove o diálogo entre as práticas matemáticas locais (êmica) com aquelas praticadas na academia (ética), pois possibilita uma aproximação desses conhecimentos por meio da elaboração de atividades matematizantes e contextualizadas no cotidiano (ROSA; OREY, 2018). 
ressaltar que oposto também pode gerar novos contextos para o desenvolvimento do conhecimento matemático.

\section{Etnomodelagem como um processo translacional ${ }^{48}$ e glocal}

A Etnomodelagem pode contribuir para a construção do conhecimento matemático glocal, pois utiliza uma variedade de referências culturais locais e globais. Por exemplo, Rosa e Orey (2017a) afirmam que essa mistura de influências produz uma identidade de grupo que busca a compreensão do conhecimento matemático de uma maneira ampla, holística e glocalizada.

Nesse contexto, é necessário que os membros de grupos culturais distintos desenvolvam uma consciência global e local em relação ao próprio conhecimento matemático por meio da tradução entre essas duas abordagens. Assim, a "tradução é um processo dinâmico de intercâmbio intercultural" (YIFENG, 2009, p. 89) que inclui a difusão, a interpretação e o compartilhamento de valores, crenças, histórias, conhecimento matemático e narrativas linguísticas, sociais, culturais e geográficas.

Esse contexto possibilita a utilização da tradução entre diferentes sistemas de conhecimento matemático para modelar práticas matemáticas desenvolvidas em culturas locais (êmicas) e globais (éticas) (EGLASH et al., 2006; ROSA; OREY, 2010) com a elaboração de etnomodelos. Nesse sentido, a tradução envolve um processo de negociação de significados e representações matemáticas que são expressas de acordo com os contextos local e global por meio da glocalização.

\section{Um Exemplo de Etnomodelagem: Métodos Antigos para a Resolução de Problemas}

Os métodos antigos que foram utilizados para resolver problemas podem ser modelados por meio da etnomodelagem. Por exemplo, os estudos das tábuas de argila babilônias fornecem uma compreensão de como os povos antigos determinaram as soluções geométricas para resolver os problemas envolvendo a área e as dimensões de retângulos e quadrados (HOYRUP, 2002). Essa abordagem propicia uma oportunidade relevante para que os educadores relacionem os eventos atuais com a importância dos artefatos culturais no contexto da Etnomatemática, da Modelagem, da história e da cultura (ROSA; OREY, 2008) por meio da Etnomodelagem.

Historicamente, esse aspecto auxiliou no desenvolvimento de uma solução geral para as equações quadráticas por meio da técnica de completar quadrados. O interesse nesse tipo

${ }^{48}$-O termo translacional está relacionado com o ato ou o processo de tradução de ideias e procedimentos matemáticos entre sistemas de conhecimento distintos, como, por exemplo, o acadêmico (CORTES, 2017). 
de resolução pode ter a sua origem relacionada com a utilização de formatos retangulares e quadrangulares para a determinação de áreas para serem irrigadas e cultivadas antes e depois das inundações provocadas pelos rios (ROSA; OREY, 2008). Por exemplo, o problema encontrado na tábua de argila YBC 6967, foi escrito no dialeto acadiano por volta de 1500 a.C., sendo estudado e editado por Neugebauer e Sachs (1945):

O comprimento de um retângulo excede sua largura em sete unidades. A área do retângulo é de sessenta unidades quadradas. Quais são o comprimento e a largura do retângulo?

A solução retórica (conhecimento matemático local e êmico) desenvolvida pelos babilônios (JOSEPH, 1991) pode ser verificada por meio da aplicação de seis etapas:

1) Determine a metade do valor pelo qual o comprimento do retângulo excede a sua largura. O resultado é $7 \div 2$, que é igual a 3,5 .

2) Multiplique 3,5 por 3,5 . O resultado é 12,25 .

3) Adicione 60 e 12,25. O resultado é $72,25$.

4) Determine a raiz quadrada de 72,25 . O resultado é 8,5 .

5) Subtraia 3.5 de 8.5. O resultado é 5 .

6) Adicione 3.5 a 8.5. O resultado é 12.

O comprimento do retângulo é de 12 unidades e a sua largura é de 5 unidades.

Esse procedimento retórico (êmico-local) que foi adotado pelos babilônios para que pudessem resolver equações quadráticas revela uma técnica simples e bem sucedida com relação à sua capacidade de desenvolver um procedimento matemático que permitisse a resolução desse problema específico. De acordo com Joseph (1991), esse procedimento direcionou os babilônios para o desenvolvimento de um método geral para a resolução de equações quadráticas.

Do ponto de vista da Etnomodelagem, a solução desse problema mostra como os babilônios geraram um conhecimento matemático sofisticado (êmico-local), que possibilitou o desenvolvimento dos procedimentos utilizados para a resolução de equações quadráticas semelhantes ao método algébrico empregado universalmente na atualidade.

Contudo, ressalta-se que esse problema babilônio também pode ser resolvido com a aplicação do conhecimento matemático acadêmico atual (global-ético). Assim, é importante considerar $C$ e $L$ como o comprimento e a largura do retângulo, respectivamente:

$$
\begin{aligned}
& \text { I) } C=L+7 \\
& \text { II) } C \bullet L=60
\end{aligned}
$$

Então, é necessário substituir a equação I na equação II. Assim, tem-se que: 


$$
\begin{aligned}
& (L+7) \bullet L=60 \\
& L^{2}+7 L=60 \\
& L^{2}+7 L-60=0
\end{aligned}
$$

Em seguida, aplica-se a fórmula quadrática49:

$$
L=\frac{-7 \pm \sqrt{49+240}}{3}
$$

Como era conhecido na maioria das civilizações antigas, os babilônios somente compreendiam e utilizavam os números positivos e, assim, determinavam apenas as raízes positivas dessas equações. Talvez os babilônios utilizassem apenas as raízes positivas, pois essas soluções adquiriram praticidade na solução dos problemas que enfrentavam no cotidiano.

É importante enfatizar que, historicamente, os números negativos somente foram aceitos como números verdadeiros apenas no século XVI (BOURBAKI, 1998). Dessa maneira, continuando com a resolução da fórmula quadrática, a seguinte equação é obtida:

$$
\begin{aligned}
& L=\frac{-7+\sqrt{289}}{2} \\
& L=\frac{-7+17}{2} \\
& L=5
\end{aligned}
$$

Finalmente, substitui-se L $=5$ na equação I.

$$
\begin{aligned}
& C=L+7 \\
& C=5+7 \\
& C=12
\end{aligned}
$$

Comparando-se as duas resoluções, observa-se que os resultados obtidos foram os mesmos, pois os métodos utilizados mostram uma estreita correspondência entre a abordagem babilônia (êmica-local) e as variantes simbólicas modernas (ética-global) (JOSEPH, 1991), que conduz a um processo dialógico de resolução para a situação-problema proposta com a utilização de etnomodelos.

Assim, para modelar essa situação-problema por meio da elaboração de um etnomodelo ético ${ }^{\circ}$, é importante estabelecer que a) a diferença entre as medidas das duas

49A denominação de Fórmula de Bháskara para a resolução de equações de segundo grau se estabeleceu no Brasil no início da década de 1960. Esse costume aparentemente brasileiro parece inadequado, pois os problemas relacionados com as equações do segundo grau eram resolvidos pelos babilônios, em escrita cuneiforme e em tábuas de argila, há quase 4 mil anos (ROSA; OREY, 2008).

50Os etnomodelos éticos são elaborados conforme a visão dos observadores externos em relação aos sistemas retirados do cotidiano (realidade local), cujas ideias, procedimentos e práticas matemáticas são modeladas (CORTES, 2017). 
dimensões do retângulo é representada pela variável $d$ e b) a área dessa figura geométrica é representada pela variável $A$.

Nesse sentido, estabelece-se que $C$ e $L$ representam o comprimento e a largura do retângulo.

$$
\begin{aligned}
& \text { I) } C=L+d \\
& I I) C \bullet L=A
\end{aligned}
$$

Então, é necessário substituir a equação $I$ na equação $I I$.

$$
\begin{aligned}
& (L+d) \bullet L=A \\
& L^{2}+L d-A=0
\end{aligned}
$$

Em seguida, é importante que a fórmula quadrática seja aplicada.

$$
\begin{aligned}
& L=\frac{-d \pm \sqrt{d^{2}-4 \bullet 1 \bullet A}}{2 \bullet 1} \\
& L=\frac{-d \pm \sqrt{d^{2}-4 A}}{2} \\
& L=\frac{-d+\sqrt{d^{2}+4 A}}{2}
\end{aligned}
$$

Substituindo-se $L$ na equação $I$, determina-se a largura do retângulo.

$$
\begin{aligned}
& C=L+d \\
& C=\frac{-d+\sqrt{d^{2}+4 A}}{2}+d \\
& C=\frac{-d+\sqrt{d^{2}+4 A}+2 d}{2} \\
& C=\frac{d+\sqrt{d^{2}+4 A}}{2}
\end{aligned}
$$

É importante reforçar que esses construtos éticos podem ser considerados como as descrições das ideias, procedimentos e práticas expressas em termos matemáticos das categorias que são consideradas significativas e apropriadas pela comunidade de observadores externos e que são fundamentais para o processo de valorização do patrimônio de culturas distintas (ROSA; OREY, 2017b).

Por outro lado, para modelar essa situação-problema, de acordo com Cortes (2017), pode-se elaborar um etnomodelo dialógico que busca traduzir uma determinada prática matemática (método babilônio de resolução de equação quadrática), que é desenvolvida pelos 
membros desse grupo cultural (êmico-local) para que os observadores externos (ético-global) possam observar e explicar essa prática matemática em termos holísticos (globalmente).

Por conseguinte, nessa tradução dialógica, a abordagem êmica busca esclarecer as distinções culturais internas à cultura enquanto a abordagem ética visa estudar a objetividade externa com relação às práticas matemáticas desenvolvidas pelos membros de um determinado grupo cultural.

Desse modo, para modelar o método babilônio de resolução da equação quadrática por meio da utilização de um etnomodelo dialógico tem-se que:

a) Para iniciar esse processo, é necessário calcular a metade da diferença entre as duas dimensões.

$$
\frac{d}{2}
$$

b) Então, eleva-se ao quadrado o resultado obtido na etapa $a$.

$$
\left(\frac{d}{2}\right)^{2}=\frac{d^{2}}{4}
$$

c) A seguir, a área $A$ do retângulo deve ser adicionada ao resultado obtido na etapa $b$.

$$
\begin{aligned}
& \frac{d^{2}}{4}+A \\
& \frac{4 A+d^{2}}{4}
\end{aligned}
$$

d) A raiz quadrada do resultado obtido no passo $c$ deve ser determinada.

$$
\begin{aligned}
& \sqrt{\frac{4 A+d^{2}}{4}} \\
& \frac{\sqrt{4 A+d^{2}}}{2}
\end{aligned}
$$

e) A largura do retângulo é determinada adicionando-se metade da diferença $d$ ao resultado obtido no passo $d$.

$$
\begin{aligned}
& C=\frac{d}{2}+\frac{\sqrt{4 A+d^{2}}}{2} \\
& C=\frac{d+\sqrt{4 A+d^{2}}}{2}
\end{aligned}
$$

f) O comprimento do retângulo é determinado pela subtração da metade da diferença $d$ no resultado obtido na etapa $e$.

$$
\begin{gathered}
C=-\frac{d}{2}+\frac{\sqrt{4 A+d^{2}}}{2} \\
C=\frac{-d+\sqrt{4 A+d^{2}}}{2} \\
\text { ISSN 2526-2882 }
\end{gathered}
$$


Esse método retórico local (êmico) utilizado pelos babilônios para resolver essa situação-problema pode ser considerado como uma derivação da fórmula quadrática, que também pode ser obtida coma aplicação do método de completar quadrados. Nesse exemplo, a glocalização pode ser entendida como a particularização do universal, que é a adaptação local por meio da tradução entre as abordagens global e local.

Esse exemplo mostra que a glocalização promove uma relação dialógica entre diferentes culturas e visões de mundo (YANG, 2003). Por outro lado, o processo de Etnomodelagem do método babilônio possibilita o entendimento sobre o funcionamento desse procedimento matemático em termos práticos. Essa perspectiva promove a visão de que os sistemas de conhecimento local podem ser incluídos no repositório global, possibilitando a geração de ambientes que promovam o diálogo entre diversos sistemas de conhecimento (ROBERTSON, 1995), como, por exemplo, os conhecimentos matemáticos local e global.

Portanto, uma utilização efetiva da Etnomodelagem pode auxiliar no estabelecimento de relações entre as estruturas conceituais do conhecimento matemático local (êmico) com as ideias, procedimentos e práticas matemáticas embutidas em projetos globais (éticos). Por exemplo, em alguns casos, a tradução de práticas matemáticas para diferentes sistemas matemáticos é direta e simples, como, por exemplo, os calendários e as práticas de contagem.

No entanto, há casos em que as ideias e os procedimentos matemáticos são incorporados nos processos de iteração encontrados nos caminhos eulerianos que estão implícitos em desenhos africanos de areia como o SONA. É importante ressaltar que, nesse ato de tradução, o conhecimento matemático pode ter uma origem êmica (local) ao invés de ética (global) (EGLASH et al, 2006; ROSA; OREY, 2010).

O problema babilônio é um exemplo do processo de glocalização que possibilitou a tradução entre as abordagens matemáticas local e global desse conhecimento. De acordo com Robertson (1995), nesse processo de translação, a glocalização captou a simultaneidade e a copresença de tendências universalizantes (global) e particularizantes (local) durante o desenvolvimento de interações culturais.

Embora as concepções eurocêntricas (globais) da Matemática tenham sido impostas mundialmente como um padrão de comportamento humano racional, é importante entender que as ideias, os procedimentos e as práticas matemáticas locais estão se espalhando rapidamente em todo o mundo (D’AMBROSIO, 2006a). Então, a glocalização propicia o desenvolvimento de um ambiente inclusivo para abordar os interesses complementares entre a globalização e a localização.

Nesse sentido, Yifeng (2009) argumenta que as linguagens universalizadas e universalizantes podem reavivar, reviver e reforçar a identificação cultural por meio da realização de atividades translacionais, que estão presentes nas realidades locais em relação ao mundo global das culturas transnacionais. Nesse contexto, a tradução desempenha um papel 
fundamental na promoção da glocalização, pois valoriza os saberes e fazeres desenvolvidos pelas culturas locais, bem como reconhece as limitações do conhecimento desenvolvido pelas culturas globais.

No processo de Etnomodelagem, a localização que se manifesta na tradução está relacionada com o ato de valorizar e projetar as culturas locais no contexto global. A cultura local está enraizada em suas tradições e, quando confrontada com uma representação global, no processo de tradução, a alteridade cultural ${ }^{1}$ é evidenciada.

Assim, a etnomodelagem visa melhorar a compreensão dos membros de grupos culturais distintos sobre a valorização das interações culturais, históricas e contemporâneas que podem ser examinadas por meio da utilização das ideias, procedimentos e práticas matemáticas através da aplicação do processo translacional entre sistema de conhecimento matemático distintos nas investigações em Etnomodelagem.

\section{Considerações Finais}

A matemática acadêmica contemporânea é predominantemente eurocêntrica e possibilitou um processo de globalização que tem dificultado ou eliminado muitas formas tradicionais de práticas matemáticas locais. Nesse contexto, as ideias e os procedimentos matemáticos estão em constante estado de negociação e interpretação, sempre mutáveis, sempre contestados e, às vezes, contraditórios e, continuamente, reposicionados pelas especificidades do espaço, do tempo, da história e das experiências e vivências culturais.

Nesse contexto, existe a necessidade de se investigar a aquisição dos conhecimentos local (êmico) e global (ético) para a implantação da Etnomodelagem como campo de pesquisa em Educação Matemática. A abordagem local (êmica) é essencial para compreensão intuitiva das ideias e dos procedimentos matemáticos de um determinado grupo cultural, sendo importante para a condução de pesquisas etnográficas enquanto a abordagem global (ética) é relevante para a comparação das práticas matemáticas desenvolvidas pelos membros desses grupos.

Desse modo, o desenvolvimento de formas sofisticadas de compreensão cultural da Matemática acompanham as aplicações da Etnomodelagem para direcionar as questões locais para uma discussão global por meio da glocalização. Essa relação dialógica entre as ideias, procedimentos e práticas matemáticas delineiam as perspectivas culturais da Etnomodelagem, pois a glocalização se aplica à combinação dessas duas abordagens que captam a proporcionalidade do local em relação ao global e vice-versa.

${ }^{51} \mathrm{~A}$ alteridade cultural é a condição de ser diferente com relação aos sujeitos ou aos objetos pesquisados, bem com aos contextos nos quais as investigações estão sendo conduzidas (ROSA; OREY, 2017b). ISSN 2526-2882

$$
\text { * } 277 *
$$


Nesse sentido, a partir de conhecimentos matemáticos desenvolvidos em contextos culturais distintos, a localização propicia a sua interação com a globalização de uma maneira dialógica. Por conseguinte, a base do processo da Etnomodelagem envolve a tradução e a interpenetração dos conhecimentos local e global, pois tem como objetivo a compreensão do dinamismo cultural resultante desse processo.

Assim, essa relação dialógica se constitui em um aspecto relevante da Etnomodelagem, pois é uma das maneiras pelas quais as culturas glocalizam as suas ideias, os seus procedimentos e as suas práticas. Por conseguinte, quando culturas se encontram e se envolvem através de interações e diálogos, as ideias, os procedimentos e as práticas matemáticas locais e universais emergem desses contextos. Portanto, o reconhecimento das semelhanças dessas práticas é importante para possibilitar a percepção de que o conhecimento local pode possuir elementos globais e vice-versa.

Desse modo, a abordagem dialógica possibilita o desenvolvimento de uma ação transformadora, pois os saberes e fazeres locais e os conhecimentos globais podem se beneficiar mutuamente (FERNANDEZ, 2009). Por exemplo, quando envolvidos em interações e diálogos, os membros de grupos culturais distintos são confrontados com ideias e procedimentos conflitantes, que os direcionam para a conscientização da utilização de estratégias e técnicas alternativas para que possam comparar, contrastar e avaliar as próprias técnicas matemáticas diárias de uma maneira crítica e reflexiva, possibilitando a exploração e a adoção de práticas matemáticas utilizadas em outras culturas.

Por conseguinte, os membros desses grupos culturais distintos podem avaliar e explorar os benefícios e os riscos das influências da globalização enquanto estão enraizados em sua própria cultura (CHENG, 2005). Nessa perspectiva, é importante que esses membros se conscientizem sobre a importância do conhecimento local para estimular e motivar a valorização da singularidade própria cultura (ROSA; OREY, 2015). Essa perspectiva assegura que as culturas locais não sejam sobrecarregadas pelas influências externas da globalização à medida que o mundo continua se interconectando rapidamente (FRIEDMAN, 2000).

Essa abordagem possibilita o desenvolvimento do compartilhamento de ideias, procedimentos e práticas matemáticas por meio de um processo denominado de continuum glocalizante $5^{2}$, no qual a globalização e a localização evoluem para um processo de conflação53 culturalmente rica, pois de acordo com Iser (1994), a traduzibilidade prova ser um contra

${ }^{52} \mathrm{~A}$ glocalização produz uma gama de processos que formam um continuum glocalizante que compreende as práticas matemáticas locais (êmicas) e as globais (éticas). Nesse continuum, a glocalização se movimenta entre esses dois extremos. Então, ao se estudar os fenômenos glocais, devese avaliar o grau de relatividade das abordagens locais e globais nesse continuum (ROSA; OREY, 2017b). 53A conflação cultural é um processo contínuo que integra as ideias, os procedimentos e as práticas matemáticas desenvolvidas pelos membros de grupos culturais distintos. Essa troca de capital cultural enriqueceu as culturas, pois conectou as vivências e as experiências que os seus membros adquirem em contextos diversos (ROSA, 2010). 
conceito à ideia predominante de hierarquia cultural dominante e colonizadora. Essa perspectiva é um aspecto importante para o desenvolvimento de investigações em Etnomodelagem.

Essa "transposição vai contra a ideia da hegemonia de uma cultura sobre a outra e, portanto, a noção de traduzibilidade surge como um contra conceito para uma sobreposição mútua de culturas" (ISER, 1994, p. 4). Assim, a tradução no processo de Etnomodelagem visa a compreensão das ideias, dos procedimentos e das práticas matemáticas utilizadas por esses membros para solucionar os fenômenos que ocorrem em seu cotidiano. Portanto, uma das características mais importantes da Etnomodelagem é o engajamento em um diálogo glocal entre as abordagens global (ética) e local (êmica) por meio do qual diversas formas de conhecimento matemático se entrelaçam.

Em concordância com esse contexto, um estudo sistemático da Etnomodelagem tem como objetivo a valorização dos fenômenos matemáticos enraizados em contextos culturais distintos, pois visa a promoção da sensibilidade cultural54 no processo de ensino e aprendizagem em Matemática. Do ponto de vista dialógico, a condução de pesquisas fundamentadas metodologicamente por essas duas abordagens pode possibilitar a obtenção de um entendimento completo e de uma compreensão ampla sobre os conhecimentos matemáticos desenvolvidos pelos membros de grupos culturais distintos.

Nesse direcionamento, a Etnomodelagem pode ser definida como o estudo de fenômenos matemáticos em uma determinada cultura, portanto, diferenciando-se da concepção de modelagem tradicional, pois é um construto social e culturalmente vinculado. Nesse processo glocalizante, o conhecimento matemático também pode se adaptar às outras visões de mundo, pois pode ser traduzido entre sistemas de conhecimentos matemáticos distintos por meio da elaboração de etnomodelos.

\section{Referências}

ANDERSON, D. Multicultural group work: a force for developing and healing. The Journal for Specialists in Group Work, v. 32, n. 3, p. 224-244, 2007.

BARBER, B. Jihad vs. me world. In: LECHNER, F. J.; BOLI, J. (Eds.). The Globalization

Reader. Malden, MA: Blackwell, 2004. pp. 29-35.

54A sensibilidade cultural está relacionada com a capacidade que os membros de grupos culturais distintos têm de se conscientizarem sobre as diferenças e semelhanças entre as culturas, sem atribuir valores culturais, como, por exemplo, positivos ou negativos, melhores ou piores e certos ou errados (ROSA, 2010). 
BASSANEZI, R. C. Ensino-aprendizagem com modelagem matemática. São Paulo, SP: Editora Contexto, 2002.

BISHOP, A. J. Mathematical enculturation. A cultural perspective on mathematics education. Dordrecht: The Netherlands: Kluwer Academic Publishers, 1988.

BOURBAKI, N. Elements of the History of Mathematics. New York, NY: SpringerVerlag, 1998.

CHENG, Y. C. New paradigm for re-engineering education. New York, NY: Springer, 2005 .

CORTES, D. P. O. Re-significando os conceitos de função: um estudo misto para entender as contribuições da abordagem dialógica da Etnomodelagem. Dissertação (Mestrado Profissional em Educação Matemática). 225 p. Instituto de Ciências Exatas e Biológicas - ICEB. Departamento de Educação MatemáticaDEEMA. Ouro Preto, MG: UFOP, 2017.

D’AMBROSIO, U. Etnomatemática: um program. A Educação Matemática em Revista, v. 1 , n. 1 , p. 5-11, 1993.

D'AMBROSIO, U. The program ethnomathematics: a theoretical basis of the dynamics of intra-cultural encounters. The Journal of Mathematics and Culture, v. 1, n. 1, p. 1-7, 2006a.

D'AMBROSIO. U. The program ethnomathematics and the challenges of globalization. Circumscribere: International Journal for the History of Science, v. 1, p. 74-82, 2006b.

EGLASH et al. Culturally situated designed tools: ethnocomputing from field site to classroom. American Anthropologist, v. 108, n. 2, p. 347-362, 2006.

FERNANDEZ, S. A. A theory of cultural glocality. Master Thesis. College of Arts and Science. Department of Philosophy. Jacksonville, FL: University of North Florida, 2009.

FREIRE, P. Educação como prática de liberdade. São Paulo: Paz e Terra, 2002. 
FRIEDMAN, T. The Lexus and the olive tree: understanding globalization. New York, NY: Random House, 2000.

HOYRUP, J. Lengths, widths, surfaces: a portrait of old Babylonian algebra and its kin. New York, NY: Springer-Verlag, 2002.

ISER, W. On translatability. Surfaces, v. 4307, 5-13, 1994.

JOSEPH, G. G. The crest of the peacock: non-European roots of mathematics. Princeton, NJ: Princeton University Press, 1991.

KHONDKER, H. H. Glocalization as globalization: evolution of a sociological concept. Bangladesh e-Journal of Sociology, v. 1, n. 2, 1-9, 2004.

KLOOS, P. The dialectics of globalization and localization. In D. Kalb, M. van der Land, R. Staring, B. van Steenbergen \& N. Wilterdink (Eds.), The ends of globalization: bringing society back in (pp. 281-298). Lanham, MD: Rowman \& Littlefield, 2000.

LATOUR, B. We have never been modern. Cambridge, MA: Harvard University Press, 1993.

NEUGEBAUER, O.; A. SACHS. Mathematical cuneiform texts. New Haven, CT: American Oriental Society, 1945

OREY, D. C. The ethnomathematics of the Sioux tipi and cone. In: SELIN, H. (Ed.). Mathematics across culture: the history of non-western mathematics. Dordrecht, The Netherlands: Kulwer Academic Publishers, 2000. pp. 239-252.

POWELL, A. B.; FRANKENSTEIN, M. (1997). Introduction. In: POWELL, A. B.; FRANKENSTEIN, M. (Eds.). Ethnomathematics: challenging eurocentrism in mathematics education. New York, NY: State University of New York Press, 1997. pp. $1-4$.

ROBERTSON, R. Glocalization: time-space and homogeneity-heterogeneity. M. FEATHERSTONE et al. (Eds.). Global modernities. London, England: Sage, 1995. pp. 25-44.

ROSA, M. A mixed-method study to understand the perceptions of high school leaders about English Language Learners (ELLs): the case of ISSN 2526-2882 $* 281 *$ 
mathematics. College of Education. Doctorate Dissertation. Sacramento, CA: CSUS, 2010.

ROSA, M.; OREY, D. C. A geometric solution for an ancient Babylonian problem. CMC ComMuniCator, v. 33, n. 2, p. 34-35, 2008.

ROSA, M.; OREY, D. C. Ethnomodelling: a pedagogical action for uncovering ethnomathematical practices. Journal of Mathematical Modelling and Application, v. 1, n. 3, p. 58-67, 2010.

ROSA, M.; OREY, D. C. Ethnomodelling as a methodology for ethnomathematics. In: STILLMAN, G. A.; BROWN, J. (Orgs.). Teaching mathematical modelling: connecting to research and practice. International perspectives on the teaching and learning of mathematical modelling. Dordrecht, The Netherlands: Springer, 2013. pp. 77-88.

ROSA, M.; OREY, D. C. A trivium curriculum for mathematics based on literacy, matheracy, and technoracy: an ethnomathematics perspective. $Z D M$, v. 47, n. 4 , p. $587-598,2015$

ROSA, M.; OREY, D. C. Influências etnomatemáticas em salas de aula: caminhando para a ação pedagógica. Curitiba, PR: Editora Appris, 2017a.

ROSA, M.; OREY, D. C. Etnomodelagem: a arte de traduzir práticas matemáticas locais. São Paulo, SP: Editora Livraria da Física, 2017b.

ROSA, M.; OREY, D. C. Etnomatemática: investigações em etnomodelagem. Revista de Investigação e Divulgação em Educação Matemática., v. 2, n. 1, p. 111-136, 2018.

ROWE, W.; V. SCHELLING. Memory and modernity: popular culture in Latin America. London, England: Verso, 1991.

SUE, D. W.; SUE, D. Counseling the culturally diverse: theory and practice. New York, NY: John Wiley \& Sons, 2003.

YANG, E. The circulation of East Asian trendy dramas grounded on cultural proximity. Bansong Yeongu, v. 69, p. 197-220, 2003. 
YIFENG, S. Cultural translation in the context of glocalization. Ariel, v. 40, n. 2-3, p. 89-110, 2009.

\section{Biografia Resumida}

Milton Rosa: Licenciado em Ciências (Curta) e Matemática (Plena) e Pedagogia, pela Faculdade de Ciências e Letras Plínio Augusto do Amaral, em Amparo, São Paulo; Especialista em Educação, Etnomatemática e Modelagem, pela Pontifícia Universidade Católica de Campinas - PUCC, em Campinas, São Paulo; Mestre em Educação Matemática, Currículo e Instrução, pela California State University, Sacramento - CSUS, em Sacramento, California, Estados Unidos; Doutor em Educação, Liderança Educacional na Educação Básica, pela California State University, Sacramento - CSUS, em Sacramento, California, Estados Unidos e Pós Doutorado, em Educação, Etnomodelagem, na Universidade de São Paulo - USP. Professor Associado I, do Departamento de Educação Matemática DEEMA, no Instituto de Ciencias Exatas e Biológicas - ICEB, da Universidade Federal de Ouro Preto - UFOP, em Ouro Preto, Minas Gerais.

Lattes: http://lattes.cnpq.br/5977637226430625.

Contato: milton.rosa@gmail.com 